\title{
CAUCHY PROBLEMS INVOLVING A SMALL PARAMETER
}

\author{
BY FRANK HOPPENSTEADT ${ }^{1}$
}

Communicated by Fred Brauer, June 17, 1969

The purpose of this note is to indicate how certain asymptotic methods developed for ordinary differential equations can be extended and applied to initial-boundary value problems for nonlinear parabolic and hyperbolic equations. This is done by considering the initial-boundary value problem as a Cauchy problem for an ordinary differential equation in an abstract space.

We consider the initial value problem

$$
\epsilon(d v / d t)-A(t, \epsilon) v=f(t, v, \epsilon), \quad 0 \leqq t \leqq T, \quad v(0)=\stackrel{i}{v}(\epsilon)
$$

where $v$ is an element of a Banach space $E$ and $\epsilon>0$ is a small parameter. The (possibly unbounded) linear operators $A$ are assumed to have a common domain of definition $\mathscr{D}$ independent of $(t, \epsilon)$, and the function $f$ is assumed to have continuous derivatives with respect to $t, \epsilon$ and continuous Fréchet derivatives with respect to $v$. Finally, $\because(\epsilon) \in D$ has continuous derivatives with respect to $\epsilon$.

We will outline here a method for finding an expansion for the solution of (1) which is valid as $\epsilon \rightarrow 0$.

1. Formal method. We begin by formally describing the procedure. These steps will be justified by Theorems $1-3$. Suppose

(I) the operator $A(t, \epsilon)$ has a bounded inverse for each $(t, \epsilon)$ and $A(t, \epsilon) A^{-1}(0,0)$ has continuous derivatives with respect to $(t, \epsilon)$.

Assuming for the moment that (1) has a solution for $\epsilon>0$, we differentiate (1) successively with respect to $\epsilon$ and set $\epsilon=0$ in the results. This gives the system of equations

$$
\begin{aligned}
-A(t, 0) v_{0} & =f\left(t, v_{0}, 0\right) \\
-\left[A(t, 0)+f_{r}\left(t, v_{0}(t), 0\right)\right] v_{r} & =R_{r}(t), \quad r=1,2, \cdots,
\end{aligned}
$$

for the coefficients $v_{r}$ of the Taylor expansion of $v$ about $\epsilon=0$. Next, we make the change of variables $t=\epsilon \tau$ in (1):

$$
d V / d \tau-A(\epsilon \tau, \epsilon) V=f(\epsilon \tau, V, \epsilon), \quad V(0)=\stackrel{\circ}{v}(\epsilon) .
$$

By differentiating this successively with respect to $\epsilon$ and setting $\epsilon=0$ in the results, we get

1 This Research was supported by the National Science Foundation under contract No. NSF GP11458. 


$$
\begin{array}{cc}
d V_{0} / d \tau-A(0,0) V_{0}=f\left(0, V_{0}, 0\right), \quad V_{0}(0)=\stackrel{\circ}{v}(0) & \\
d V_{r} / d \tau-\left[A(0,0)+f_{r}\left(0, V_{0}, 0\right)\right] V_{r}=\rho_{r}(\tau), & V_{r}(0)=\stackrel{\circ}{r}_{r}, \\
r=1,2, \cdots,
\end{array}
$$

for the coefficients $V_{r}$ of the Taylor expansion of the solution of (3) about $\epsilon=0$. In (4b) $\stackrel{\circ}{r}_{r}$ is the coefficient of $\epsilon^{r}$ in the Taylor expansion about $\epsilon=0$ of $i(\epsilon)$. We observe that for each $r, R_{r}$ in (2b) depends only on $t, v_{0}, \cdots, v_{r-1}$, and $\rho_{r}$ in (4b) depends only on $\tau, V_{0}, \cdots$, $V_{r-1}$.

If problems (2), (4) can be solved successively for the $v_{r}, V_{r}$, we can form the (possibly divergent) expansions

$$
\sum_{r=0}^{\infty} v_{r}(t) \epsilon^{r}
$$

$$
\sum_{r=0}^{\infty} V_{r}(t / \epsilon) \epsilon^{r}
$$

From analogy with the ordinary differential equations case $(A(t, \epsilon)$ bounded operators) the solution of (1) is expected to be represented by (5a) for $t$ away from zero and by (5b) for $t$ near zero. To obtain an expansion for the solution of (1) valid uniformly for $0 \leqq t \leqq T$, we employ a matching device. We observe that the expansion

$$
\sum_{r=0}^{\infty} v_{r}(\epsilon \tau) \epsilon^{r}
$$

formally satisfies the problem (3). Expanding each $v_{r}(t)=\sum_{q=0}^{\infty} v_{r, q} t^{q}$ in its formal Taylor expansion and substituting these into (6) gives

$$
\sum_{r=0}^{\infty} U_{r}(\tau) \epsilon^{r}, \quad U_{r}(\tau)=\sum_{q=0}^{r} v_{r-q, q} \tau^{q}, \quad r=1,2, \cdots
$$

It is shown below that expansion (7) is like expansion (5a) for $t$ near zero and like (5b) for $t$ away from zero. We thus arrive at the expansion

$$
\sum_{r=0}^{\infty}\left[v_{r}(t)+V_{r}(t / \epsilon)-U_{r}(t / \epsilon)\right] \epsilon^{r}
$$

Expansion (8) is considered in three different cases:

(i) Abstract Parabolic Case where for each $(t, \epsilon) \in[0, T] \times\left[0, \epsilon_{0}\right]$, $-A(t, \epsilon)$ is the infinitesimal generator of an analytic semigroup of operators in $E$ (see Kato [1]).

(ii) Abstract Hyperbolic Case where for each $(t, \epsilon),-A(t, \epsilon)$ is the infinitesimal generator of a semigroup of class $C_{0}[2]$.

(iii) Parabolic Case where $A$ is a positive definite elliptic operator in $E=L^{\infty}$. 
2. Assumptions. We assume:

(II) The problem (2a) has an isolated solution which is infinitely differentiable for $0 \leqq t \leqq T$ and $f_{v}\left(t, v_{0}(t), 0\right)=0$ for $0 \leqq t \leqq T$ (i.e., $A$ accounts for the linearization of (1) about $\left.v=v_{0}(t)\right)$.

(III) The problem (4a) has a unique solution $V=V_{0}(\tau)$ which exists for $0 \leqq \tau<\infty$.

Finally, a crucial condition for our work is:

(IV) The resolvent set of $-A(t, \epsilon)$ includes the half plane $\{\operatorname{Re} Z \geqq-\delta\}$ for some $\delta>0$.

3. Results. The proofs of the following theorems will be given elsewhere.

Theorem 1 (Abstract Parabolic Case). Let conditions (I)-(IV)

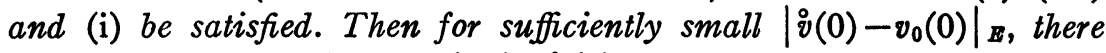
exists a unique solution $v=v(t, \epsilon)$ of (1) for each small $\epsilon$. Also, the problems (2) and (4) can be solved successively and

$$
v(t, \epsilon) \tilde{E} \sum_{r=0}^{\infty}\left[v_{r}(t)+V_{r}(t / \epsilon)-U_{r}(t / \epsilon)\right] \epsilon^{r}
$$

where $v_{r}, V_{r}, U_{r}$ are determined from (2), (4) and (7), respectively.

REMARKS. The notation $g(t, \epsilon)_{\tilde{E}} \sum_{r=0}^{\infty} \alpha_{r}(t, \epsilon) \epsilon^{r}$ here means that for each $N=1,2, \cdots$, the function $S_{N}$ defined by $\epsilon^{N+1} S_{N}=g(t, \epsilon)$ $-\sum_{r=0}^{N} \alpha_{r}(t, \epsilon) \epsilon^{r}$ is bounded in the norm of $E$ uniformly for $0 \leqq t \leqq T$, $0<\epsilon \leqq \epsilon_{0}$.

The restriction on $\left|\check{v}(0)-v_{0}(0)\right|_{z}$ in Theorem 1 is primarily to ensure that $\left|V_{0}(\tau)-v_{0}(0)\right|_{E \rightarrow 0}$ as $\tau \rightarrow \infty$. Thus, its size depends on the nonlinearity $f$ and the location of the spectrum of $-A(t, \epsilon)$.

Theorem 2. (Abstract Hyperbolic Case). Let conditions (I)-(IV) and (ii) be satisfied. Also, suppose $|A(t, \epsilon)-A(s, \epsilon)|_{E} \leqq C|t-s|$ for some constant $C>0$ (independent of $\epsilon$ ) and all $0 \leqq t, s \leqq T$. Then the conclusion of Theorem 1 remains valid.

REMARKS. An important restriction imposed by the continuity condition on $A$ in Theorem 2 is that the operator defined by the difference is a bounded operator. This condition does not appear in the parabolic case because of certain properties of analytic semigroups.

The proof of Theorems 1 and 2 rests on obtaining an estimate for the fundamental solution of problem (1). In particular, we have

LEMMA. Let either the hypotheses of Theorem 1 or 2 be satisfied. Then 
for each $\epsilon>0$, there is a fundamental solution, $U(t, s, \epsilon)$, for the linear part of problem (1) (i.e. (1) with $f=0$ ). Moreover there are positive constants $K, \eta$ such that

$|U(t, s, \epsilon)|_{E} \leqq K \exp [-\eta(t-s) / \epsilon] \quad$ for $0 \leqq s \leqq t \leqq T, \quad 0<\epsilon \leqq \epsilon_{0}$.

The proof of the following theorem rests on obtaining a similar estimate for the fundamental solution when $E=L^{\infty}$. Let $\Omega$ be a bounded domain in Euclidean $n$-space $E^{n}$ with boundary $\partial \Omega$ and closure $\bar{\Omega}$. A point $x \in E^{n}$ is given by $x=\left(x_{1}, \cdots, x_{n}\right)$, and we use the notation $D_{i}=\partial / \partial x_{i}$. We shall denote by $\mathfrak{A}(x, t, \epsilon, D)$ a second order linear differential operator in $L^{\infty}$ with real coefficients:

$$
\mathfrak{A}(x, t, \epsilon, D)=\sum_{i, j=1}^{n} a_{i j}(x, t, \epsilon) D_{i} D_{j}+\sum_{i=1}^{n} a_{i}(x, t, \epsilon) D_{i}+a(x, t, \epsilon) .
$$

The coefficients of $\mathfrak{A}$ have continuous derivatives of all orders with respect to $(x, t, \epsilon) \in \bar{\Omega} \times[0, T] \times\left[0, \epsilon_{0}\right]$. Also, the matrix $\left(a_{i j}\right)$ is symmetric and positive definite uniformly in $(x, t, \epsilon)$ (in particular at $\epsilon=0$ ). Finally, $\Omega$ is of class $C^{2}$.

Consider the initial-boundary value problem

$$
\begin{aligned}
\epsilon u_{t}-\mathfrak{A} u & =f(x, t, u, \epsilon), \quad u=0 \text { on } \partial \Omega \times[0, T], \\
u(x, 0, \epsilon) & =\grave{u}(x, \epsilon) \text { on } \bar{\Omega} .
\end{aligned}
$$

Here $u_{t}$ denotes $(\partial u / \partial t)$, and we assume $f, i$ have continuous derivatives of all orders. The formal considerations above proceed in the same way for this problem. We will denote by $\left(2^{*}\right),\left(4^{*}\right)$, etc., those statements reinterpreted for (9). We then have

Theorem 3 (Parabolic CASE). Let $\mathfrak{A}, f, \mathfrak{u}$ be as above and let condition $\left(\mathrm{II}^{*}\right)-\left(\mathrm{IV}^{*}\right)$ be satisfied. Then for $\left|\dot{u}(x, 0)-v_{0}(x, 0)\right|_{L^{\infty}}$ sufficiently small, there is a unique solution of (9) for each small $\epsilon$. Moreover, $\left(2^{*}\right),\left(4^{*}\right)$ can be solved successively and

$$
u(x, t, \epsilon) \tilde{\mathrm{L}}^{\infty} \sum_{r=0}^{\infty}\left[v_{r}^{*}(x, t)+V_{r}^{*}(x, t / \epsilon)-U_{r}^{*}(x, t / \epsilon)\right] \epsilon^{r} .
$$

REMARKs. In (9), $\mathfrak{A}$ can be replaced by an elliptic operator of order $2 m$ for any integer $m>0$ with a corresponding change in the boundary conditions (see e.g., Agmon [3]). The estimate in $L^{\infty}$ for the fundamental solution of problem (9) is obtained from the maximum principle for parabolic equations.

In [4] Keller formally ootained an expansion for the solution of (9) in the linear case (i.e., $f$ independent of $v$ ). His expansions involved 
the eigenfunctions of $\mathfrak{A}$. Since the Green's function for the linear part of (9) can be expanded in terms of the eigenfunctions of $\mathfrak{A}$, the expansion of Theorem 3 can be given in terms of these eigenfunctions. In the linear case the result agrees with that in [4].

The method outlined here is essentially the one developed by Vasil'eva [5] for ordinary differential equations. Her work suggests that these methods can be extended to treat systems of the form $u_{t}=g(t, u, v, \epsilon), \epsilon v_{t}=A(t, \epsilon) v+f(t, u, v, \epsilon)$. Such extensions are presently being investigated. Finally, this work has been applied to problems involving the heat equation with nonlinear source in a domain with a slowly moving boundary.

\section{REFERENCES}

1. T. Kato, Nonlinear evolution equations in Banach spaces, Proc. Sympos. Appl. Math., vol. 17, Amer. Math. Soc., Providence, R. I., 1965, pp. 50-67. MR 32 \#1573.

2. E. Hille and R. S. Phillips, Functional analysis and semigroups, rev. ed., Amer. Math. Soc. Colloq. Publ., vol. 31, Amer. Math. Soc., Providence, R. I., 1957. MR 19, 664.

3. S. Agmon, On the eigenfunctions and on the eigenvalues of general elliptic boundary value problems, Comm. Pure Appl. Math. 15 (1962), 119-147. MR 26 \#5288.

4. J. B. Keller, Lecture notes, Math. Dept., Michigan State University, East Lansing, Mich., 1968.

5. A. B. Vasil'eva, Asymptotic behavior of solutions of certain problems for ordinary non-linear differential equations with a small parameter multiplying the highest derivatives, Uspehi Mat. Nauk 18 (1963), no. 3 (111), 15-86= Russian Math. Surveys 18 (1963), 13-84. MR 28 \#1363.

Courant Institute of Mathematical Sciences, New York University, NEW YoRK, NEW YORK 10012 